\title{
Utilizing Electronic Reading Device (Kindle) in English as Foreign Language Reading Class*
}

\author{
Min-Hsun Chiang \\ Tunghai University, Taichung, Taiwan
}

\begin{abstract}
With the evolutionary change in information and communication technologies (ICT), the definition of literacy has undergone substantial change to include the skills and strategies required to successfully adapt to the rapidly changing ICT contexts. This new definition presents an urgent need to acknowledge the gap between the types of reading experiences students come across at school and those they regularly practice outside the school. One way to bridge such gap is to expand the reading instruction to incorporate electronic reading device, like Kindle, to engage students in electronic reading. Many e-books feature multimodal functionalities, including audio, video, hyperlinks, and interactive tools. Research on this form of reading is still in its infancy in spite of some positive prospects for fostering literacy development and reading comprehension. Therefore, adopting a mixed method approach with 36 college freshmen in central Taiwan, the current study intended to fill in the void by investigating any difference in readers' reading comprehension and reading motivation, between the Kindle group and the print-based group. The results indicate that embedding aspects of technology into a traditional reading instruction boosted the participants' motivation to read. Nevertheless, the novelty-driven enthusiasm for electronic reading might not sustain students' reading motivation long enough to cultivate long-lasting reading habits. The results indicate that there was no significant difference in reading comprehension between two groups. Implications for utilizing electronic readers in EFL classes are also provided.
\end{abstract}

Keywords: electronic book (e-book), extensive reading, EFL reading instruction, reading comprehension, reading motivation

\section{Introduction}

Technological innovation has changed the way most people read newspapers, magazines, and mails. On a daily basis, more and more of our reading is from screens, or from some version of electronic reading tablets or mobile technology, instead of reading from print. There is absolutely no doubt that the definition of "book" is fundamentally and dramatically changing. Nevertheless, the definition of electronic-books (e-books) ranges from converting print-based books directly into digital form to an interactive, multimedia, online, and hyperlink embedded, portable reading device. Over the years, electronic reading environments have received increasing attention as educational tools for promoting reading fluency and enhancing reading comprehension, particularly for students who experience difficulties in learning to read and reading to learn. Many e-books feature

\footnotetext{
* Acknowledgements: This research inquiry was made possible with the funding from National Science Council, research grant number NSC 99-2410-H-029-055.

Min-Hsun Chiang, associate professor, Ph.D., Department of Foreign Languages and Literature, Tunghai University.
} 
multimodal functionalities, such as audio, video, hyperlinks, and interactive tools. These interactive tools allow readers to literally interact with the text via inserting/deleting/replacing text; marking passages by underlining/highlighting; inserting comments by attaching files/appending notes; and manipulating the text formatting on the screen page (Larson, 2009). The dynamic nature of the hypertext environment gives it an ability to find common ground with the readers who are constantly staying on a loop of texting, messaging, and facebooking.

Not everyone applauds this change in reading. The hypertext environment offers a medley of sights, sounds and links that the conventional text cannot provide. Paradoxically, the ability to provide sound, animations, video clips and the like can also be distracting for learners (Harper, Squires, \& McDougall, 2000). What is an advantage for some may be a drawback for others. Research in this area is much needed to shed light on the potential advantages and threats of electronic reading to reading engagement, reading comprehension, and reading motivation. Therefore, this study intended to investigate how the different reading modes affect subsequent reading comprehension and reading motivation. The following questions guided the research and data analysis for this study: (1) Is there any difference between the Kindle and print-based book readers in their reading comprehension after a semester long participation in Guided Reading class? (2) Is there any difference between the Kindle and print-based book readers in their reading motivation after a semester long participation in Guided Reading class? and (3) How was Kindle received by the participants? What are the participants' perceptions of utilizing Kindle in their Guided Reading class?

\section{Review of Related Work}

\section{Computer-Based Reading Environments: Advantages and Disadvantages}

The dynamic nature of the hypertext environment gives it an ability to connect with the net-generation readers. Computer environments can offer a medley of sights, sounds, and links that linear text cannot provide. Potential benefits of using technology in educational settings include a greater variety of instructional methods, potential to accommodate different learning styles and instructional needs and interactivity. Meanwhile, computer-based learning has the potential to provide the learner with a sense of agency and ownership in the process, promote intrinsic motivations for learning, offer a sense of privacy, and give immediate feedback (Leu, 2000; Meyer \& Poon, 1997; Wishart, 2000). In addition, simply adding sound to text has enormous benefits for poor readers, the hearing impaired, and children with reading disabilities (Alexander, Kulikowich, \& Jetton, 1994; Beck \& McKeown, 1999; D. E. Hailey \& C. Hailey, 1998), as the additional auditory input helps readers fill in the gaps and improve reading skills.

Paradoxically, the ability to provide sound, animations, video clips and the like can also be distracting for learners (Harper et al., 2000). What is a benefit for some may be a disadvantage for others; novice computer users have little or no prior knowledge of the technology and/or strategies that can be used to help keep them on reading track. Additionally, some learners may have a poor self-efficacy in regard to computer skills. Therefore, more research needs to be done to explore whether the computer-based reading affects readers' motivation to keep reading. Any learning environment should be accessible to diverse learning styles, be gender-neutral, and create opportunities for ownership and collaboration (Campbell, 2000). Some may believe that no crucial differences exist between the readings of print and electronic text given the fact that both involve the processes of word recognition, prior knowledge activation, and meaning construction. Computer-based reading environments, nevertheless, have the potential to change the nature of reading. The important question is, "are 
these environments, better, worse, or just different from learning in more traditional environments?" No conclusive answer to this question could be drawn yet due to the fact that much of the published research did not take into account the impacts of immediate social contexts on the reader. Evidently, research in this area is much needed to shed light on the potential advantages and threats of the computer-based reading to reading comprehension and reading motivation, which constitutes some of the most important areas to study when e-book reading is concerned.

\section{Electronic Reading and Reading Comprehension}

The ultimate goal of reading is to comprehend a written text regardless of the text medium. Comprehension is, in part, hinged upon the reader's activation of prior knowledge and motivation as the author's words are read. During comprehension, readers associate the new information communicated by the text to information already in their minds (Tompkins, 2006). However, how does the difference between the electronic text and the print-based text affect the comprehension processes?

Opportunities for interacting with traditional print texts are restricted by their linear composition, and static traditional texts rely heavily on the reader's internal strategies to activate prior knowledge. Traditional texts are passive and cannot respond to individual readers. Computer-based reading, nevertheless, may involve a literal interaction between the reader and the text (Reinking, 1998). Software programs embedded in digital reading device offer a greater repertoire of comprehension cues and a fuller context for the story than traditionally printed texts (Pearman \& Lefever-Davis, 2006; Reinking, 1998). Interactive electronic storybooks enable readers with diverse backgrounds and varying reading levels to take part in a learning environment where a wider spectrum of individual needs can be met (Chu, 1995).

However, the same electronic text features that enhance context and activate prior knowledge may be detrimental to reading comprehension in the long run by allowing readers to use the features without developing competence in detecting context indicators and learning to consciously make connections between text and their own prior knowledge. Many of the software programs allow students to have words, sentences, pages, or the entire book read to them. Many also play music, contain animations, or recite the names of objects as they are selected. Some researchers have flagged these features as possible concerns (Labbo et al., 2003; Lewin, 1996). Readers may rely on the computer to decode words or to read the story rather than developing their own abilities. Moreover, if the reader does not ask for help, the computer does not offer it or provide instruction in reading strategies. Some are concerned that hypertext features allowing readers to explore options leads to superficial learning. Traditional texts encourage the reader to think critically and to bring analytical skills to bear to gain meaning while a hypertext environment fosters reliance on the technology to gain understanding. For instance, this type of superficial learning was exhibited by young readers who requested the pronunciation or definition of the same word over and over without acquiring the ability to recognize the word when it appeared repetitively in the story (Lefever-Davis \& Pearman, 2005).

A recent review of research into literacy and technology indicates the scarcity of related work that investigates if the utilization of e-reading does or does not achieve the intended outcome. The present study, therefore, aims to address this issue by investigating how EFL students' e-reading influences reading motivation and how the changes in reading motivation might affect reading comprehension, or vice versa.

\section{Motivation in Computer-Mediated Learning Environment}

It is generally agreed that computer-based activities can increase intrinsic motivation by allowing students 
to customize their work and increase their control over the task with enticed curiosity and challenge. At-risk populations may also experience an increase in self-motivation and self-interest while immersing in computer-mediated learning environments that provide graphics, sound, and other special effects, although some of these multi-sensory may be more extrinsically motivating, or even confusing for some learners. Some computer-based educational programs featuring fun-packed, arcade-like atmosphere (Kamil, Intrator, \& Kim, 2000) may provide additional advantages in promoting reader motivation and interest. According to Chan and Ahern (1999), computer-based environments facilitate learning and achievement, and also have the potential to impact the emotion and attitude of the learner. By providing motivation and situational interest, learning becomes less arduous and more fulfilling. Therefore, computer-based instructional tools have the potential to provide active engagement of the reader, the ability to find immediate definitions for unknown words, and a "motivational bounce" (Kamil et al., 2000, p. 779) needed to nudge students in the right direction.

\section{Motivation to Read in Second/Foreign Language}

Compared with a substantial body of literature in L1 reading motivation, relatively little research on L2 reading motivation in EFL settings can be found. In the field of SLA, based on expectancy-value theory, Day and Bamford (1998) provided a theoretical model of L2 reading motivation which is made up of four major components: materials, reading ability, attitudes, and sociocultural environment, including family and friends. Materials and reading ability are concerned with the expectancy construct of successful reading, whereas attitudes and sociocultural environment are related to values. Day and Bamford (1998) further argued that an individual's decision to read in a second language is strongly influenced by materials and attitudes. Unfortunately, as stated by Mori (2002) and Takase (2005), little empirical evidence has been gathered to support Day and Bamford's hypothesis.

Concerning the growing importance placed on research in foreign language teaching during the last two decades, there is a need to investigate what motivational components contribute most closely to foreign language reading motivation. Drawing upon Wigfield and Gutherie's reading motivation theory, Mori (2002) developed a reading motivation questionnaire based on Motivation to Read Questionnaire (MRQ) and administered it to 447 students at a women's university in Japan to investigate the possible components of L2 reading motivation in EFL context. In Mori's questionnaire, three dimensions, Competition in Reading, Reading in Recognition, and Social Reasons for Reading, proposed by Wigfield and Gutherie, were eliminated because they were considered to be irrelevant to the participants. Instead, Mori incorporated Gardner's concept of Integrative Orientation into her questionnaire. According to Gardner's (1985) Socio-Educational Model of Language Leaning, integrative motivation refers to individuals' desire to learn a second or a foreign language because they want to interact with target language speakers. Up to this point, contradictory results were found in numerous studies conducted in different ESL or EFL contexts: Some have shown that integrative motivation plays a key role in L2 language learning, some did not find the significance of integrative motivation. Mori included the construct of integrative motivation in her questionnaire and examined whether integrative motivation is a distinct component in the field of $\mathrm{L} 2$ reading in an EFL setting.

Mori's work (2002) revealed that there are four major components of L2 reading motivation: Intrinsic Value of Reading in English, Extrinsic Utility Value of Reading in English, Importance of Reading in English, and Reading Efficacy. These results are consistent with Wigfield and Gutherie's claim that reading motivation is multifaceted. Furthermore, Mori (2002) concluded that "foreign language reading motivation closely 
resembles more general forms of motivation as laid out in expectancy-value theory" (p. 102). Concerning integrative motivation, the results of Mori's study were consistent with other motivational research (Chen, Warden, \& Chang, 2005; Crookes \& Schmidt, 1991; Dörnyei, 1990; Kraemer, 1993) conducted in EFL contexts and did not to find the significance of integrative motivation as Gardner suggested. However, given the globalization trend, it is very likely that the English major freshmen, who will be the potential participants of the current project, would have the desire to integrate with the target language culture. Therefore, the integrative aspect of the motivation to read English as foreign language will be included in the questionnaire for this study.

\section{Research Method}

\section{Design}

A mixed-method approach was used to collect both quantitative and qualitative data from two reading classes of a total of 36 English-major students at a four-year college in central Taiwan. Quantitative data were collected from English Reading Comprehension Test (ERCT) and the Reading Motivation Questionnaire (RMQ). Qualitative data were gathered from the open-ended survey and interviews with the participants. Thirty-six students from both reading classes were randomly assigned to an experiment group with e-books (Kindle) and a control group with print-based books. In other words, in one class, half of the students were reading e-books with supported text while the other half were reading traditional books. Both groups read the same five titles of the novels. To compensate for those students in control groups who did not get the chance to read from the handheld electronic reading device, the opportunity to use Kindles during the summer vocation was offered.

\section{Pedagogical Context for the Current Project}

As aforementioned, this study was conducted with 36 freshmen from two Guided Reading classes which is a required course for English-major students at one university in central Taiwan. The class meets two periods a week. The goal of this Guided Reading course is to help students become better readers through reading intensively (following specific reading strategies and skills) and reading extensively (large amounts of authentic materials). The objective of this course is to build up reading competence by use of the following reading strategies: skimming, scanning, previewing, predicting, picturing, relating, monitoring, recalling, guessing vocabulary from context clues, recognizing word parts, main ideas versus minor details, summarizing, outlining, examining organizational patterns, making inferences, synthesizing information, distinguishing facts from opinions, and analytical reasoning. As part of the requirement, the students in the Guided Reading Class usually read six to eight authentic juvenile titles.

\section{Participants}

As a result of convenient sampling, this project recruited 36 students taking Guided Reading course taught by the researcher. According to the university record, the general English proficiency of the incoming English-major freshmen is at approximately 50 percentiles of the freshmen nationwide. However, their reading comprehension skill and computer literacy may vary from one student to another. Most of them did not have prior experience reading from Kindle. Therefore, an induction session to Kindle was offered to the participants to orient them to this electronic reading device with a hope that they can take full advantage of the multiple functions provided by this innovative reading device. 


\section{Data Collection and Analysis}

This study aimed to explore the effects of using Kindle versus print-based novels on college freshmen's reading comprehension and reading motivation. Data were gathered from the following three means: the English Reading Comprehension Test (ERCT), the Reading Motivation Questionnaire (RMQ), and an open-ended survey. RMQ is a 50-item, four-point Likert scale questionnaire, which was administered to 32 freshmen enrolled in the Guided Reading course to investigate students' foreign language reading motivation. The internal consistency estimate of reliability for the questionnaire was calculated, and Cronbach's Alpha was 0.88 .

At the outset of the study, an Independent Samples t-test was employed to find out if there was any significant difference in the participants' reading comprehension between the two groups (Kindle versus Print-Based). At the end of the study, a Paired Samples t-test was performed with the pre- and post-scores from the RMQ. Independent Samples t-test was carried out to discover if there was any significant difference in reading motivation between the two groups after the study. In addition to reading motivation, participants' preand post- scores from the ERCT were entered into SPSS 13.0 with the significant level set at $\alpha<0.5$. Two Paired Samples t-tests were conducted to see whether there were any differences in the students' reading comprehension after taking part in Guided Reading class. Besides quantitative data, written responses from the open-ended survey were coded and analyzed to tap into the participants' perception of using Kindle in their reading class.

\section{Results and Discussions}

The results will be presented as potential answers to the three research questions posted at the outset of the current study. Centering around three major findings, the results will be reported and discussed. These findings include whether there is any difference in English reading comprehension between the two groups, whether there is any difference in English reading motivation between the two groups and how the electronic reading device was received by the participants.

(1) No significant difference was identified in English reading comprehension between the Kindle group and the print-based group.

To investigate the effects of using Kindle on English reading comprehension, the mean scores of the preand post-ERCT from the Kindle and the print-based groups were first presented in Table 1. The table shows that for the print-based group, their mean score from the post-ERCT is higher than that from their pre-ERCT. It can be inferred that the participants in the print-based group made progress in their English reading comprehension after taking part in a semester-long Guided Reading class. However, for the Kindle group, their mean scores of the post-ERCT declined after a semester. Independent samples t-test was conducted to examine if there is any significant difference between the Kindle group and the print-based group in their post-ERCT scores. The result indicates no significant difference between the two groups in their ERCT posttest $(\mathrm{t}=1.1, \mathrm{p}=0.27)$. In other words, although the print-based group was found to make progress in English reading comprehension, Table 2 illustrates that there is no significant difference in ERCT between the two groups. 
Table 1

Means and Standard Deviations of the Kindle Group's and the Print-Based Group's Responses to ERCT Before and After Study

\begin{tabular}{lllll}
\hline & \multicolumn{2}{c}{ Pre-test } & Post-test \\
\cline { 2 - 5 } & Mean & Std. deviation & Mean & Std. deviation \\
\hline Kindle group & 60.35 & 11.53 & 57.47 & 10.96 \\
Print-based group & 62.41 & 12.88 & 63.06 & 17.88 \\
\hline
\end{tabular}

Table 2

Independent Samples T-Test of the Kindle and Print-Based Groups' Posttest for ERCT

\begin{tabular}{lllllll}
\hline & $\mathrm{t}$ & $\mathrm{df}$ & Sig. (2-tailed) & Mean difference & Std. error difference \\
\hline Post & Equal variances assumed & -1.10 & 34 & 0.28 & -5.59 & 5.07 \\
\hline
\end{tabular}

Table 3

Comparison of the Paired Samples T-Test Scores From the ERCT Between the Kindle and the Print-Based Groups

\begin{tabular}{|c|c|c|c|c|c|c|c|}
\hline & & Mean & Std. deviation & Std. error mean & $\mathrm{t}$ & $\mathrm{df}$ & Sig. (2-tailed) \\
\hline Kindle & Pre - Post & -2.88 & 13.75 & 3.34 & 0.86 & 17 & 0.40 \\
\hline Print-based & Pre - Post & 0.65 & 16.03 & 3.89 & -0.17 & 17 & 0.87 \\
\hline
\end{tabular}

Besides the between-group difference, the within-group differences were also examined with both groups. The paired samples t-tests with the Kindle and the print-based groups show that for both groups, there were no significant differences in their English reading comprehension before and after the project, as displayed in Table 3. Despite the fact that the mean score of the post-ERCT form the print-based group increased slightly, no conclusion can be drawn that either reading mode tends to help improve reading comprehension.

Dissimilar to the previous studies that considered digital reading devices more helpful in facilitating reading comprehension (Pearman \& Lefever-Davis, 2006; Reinking, 1998), the findings from the current study indicate that there is no difference in aiding reading comprehension whether the participants use Kindle or not. Instead, a slight decline in the post reading comprehension scores among the Kindler readers serves as a reminder that a hypertext environment may foster the readers' reliance on the technology to gain understanding (Lefever-Davis \& Pearman, 2005). In other words, a hypertext environment may prevent EFL readers from bringing analytical skills to gain meaning from the text. Consequently, their reading comprehension may be impeded.

(2) No significant difference was found between the two groups, Kindle versus Print-Based, in their post-RMQ scores.

Thirty-six participants' pre- and post- scores from the RMQ were calculated to find out if there was any difference between the electronic book and print-based book readers in their reading motivation after a semester long intervention. The mean scores from the pretest and posttest of RMQ indicate a slight increase in the Kindle group (Table 4). Independent samples t-test was conducted to examine if there is any significant difference between the Kindle group and the print-based group in their post RMQ scores. The result indicates no significant difference between the two groups in their RMQ posttest $(t=1.1, p=0.27)$. That is to say, 
although there is an increase in the Kindle group's post RMQ mean score, no statistic significant difference was identified in their reading motivation after a semester-long experiment. Table 5 illustrates that there is no significant difference in RMQ between the two groups.

Table 4

Comparisons of Kindle Group and Hard-Copy Group's Results to the Pre- and Post-Reading Motivation Questionnaire (RMQ)

\begin{tabular}{lllll}
\hline & \multicolumn{3}{c}{ Pre-test } & Post-test \\
\cline { 2 - 5 } & Mean & Std. deviation & Mean & Std. deviation \\
\hline Kindle group & 145.12 & 13.60 & 149.24 & 14.75 \\
Print-based group & 144.11 & 14.71 & 142.35 & 15.71 \\
\hline
\end{tabular}

Table 5

Independent Samples T-Test of the Kindle and Print-Based Groups' Post-test for RMQ

\begin{tabular}{|c|c|c|c|c|c|c|}
\hline & & $\mathrm{t}$ & df & Sig. (2-tailed) & Mean difference & Std. error difference \\
\hline Post & Equal variances assumed & -1.10 & 34 & 0.28 & -5.59 & 5.07 \\
\hline
\end{tabular}

On top of the between-group difference, the within-group differences were also examined with both groups. The paired samples t-tests of RMQ with the Kindle and the print-based groups show that for both groups, there were no significant differences in their English reading motivation before and after the project, as displayed in Table 6. Despite the fact that the mean score of the post-ERCT form the Kindle group increased, it remains inconclusive whether the utilization of Kindle has the potential to boost up reading motivation among EFL college students.

Table 6

Comparison of the Paired Samples T-Test Scores From the RMQ Between the Kindle and the Print-Based Groups

\begin{tabular}{lllllll}
\hline & Mean & Std. deviation & Std. error mean & $\mathrm{t}$ & $\mathrm{df}$ & Sig. (2-tailed) \\
\hline Kindle & 4.12 & 11.55 & 2.80 & 1.47 & 17 & 0.16 \\
Print-based & -2.58 & 10.28 & 2.49 & -1.04 & 17 & 0.32 \\
\hline
\end{tabular}

It is interesting to note in Table 4 that a slim decline was identified with the print-based group in their RMQ mean score after a semester-long participation in the reading class. The increase in the Kindle group's motivation to read may be attributed to learner autonomy (Guthrie, 2009) and text support (Day \& Bamford, 2002) made available by the Kindle in which the readers have more control over their reading and access to a dictionary. As a result, the participants became more motivated to read.

Table 7

Comparison of the Paired Samples T-Test Scores From the Aesthetic Enjoyment Dimension of the RMQ Between the Kindle and the Print-Based Groups

\begin{tabular}{|c|c|c|c|c|c|c|}
\hline & \multicolumn{3}{|c|}{ Paired differences } & & \multirow{2}{*}{ df } & \multirow{2}{*}{$\begin{array}{l}\text { Sig. } \\
\text { (2-tailed) }\end{array}$} \\
\hline & Mean & Std. deviation & Std. error mean & & & \\
\hline Kindle post-test - & 0.25 & 0.46 & 0.11 & 2.27 & 17 & $\begin{array}{l}0.04 \\
* *\end{array}$ \\
\hline Hard copy post-test $-\quad$ pre-test & 0.06 & 0.64 & 0.15 & 0.38 & 17 & 0.71 \\
\hline
\end{tabular}


In addition to calculating the paired-samples t-tests for the overall reading motivation, 20 paired-samples t-tests were carried out for both the Kindle and the hardcopy group to identify any significant difference across 10 subcategories of reading motivation. Only one significant difference was identified with the Kindle group in aesthetic enjoyment dimension of reading, which refers to the pleasure gained from reading a well-written book or article on a topic one finds interesting. This notion of motivation might sound similar to intrinsic motivation to read; however, this type of aesthetic enjoyment is unique to reading and differs from traditional views of intrinsic motivation. Table 7 indicates that a significant difference in the pre- and post-scores of aesthetic enjoyment of reading with the Kindle group, but not with the hardcopy group. This discrepancy brings to our mind that the Kindle helped the current participants enhance their enjoyment of experiencing various kinds of literary texts. Superior to the print-based books, the Kindle has the capacity of hosting a plethora of literary texts (Kamil et al., 2000), which also broadens the reader's spectrum of reading enjoyment.

(3) Only a fraction of the participants were receptive to reading novels on a Kindle. Majority of the participants from both groups prefer to read print-based books when they have a choice.

To the researcher's surprise, only a fraction of the participants were receptive to reading novels on a Kindle. The resistance factors are both psychological and physical. Those who were held responsible for the Kindle they possessed temporarily felt it was burdensome to own a Kindle and were afraid of losing or damaging it. Also, the technical aspects of the device hindered the full embracing of the digital native readers. Several participants complained that without thumbing through the pages kinesthetically and sniffing the book smell, they did not feel they were actually reading. The ritual of paper-based reading is irreplaceable. Participants from Kindle group pointed out other disadvantages, such as black/white screen, hard to press button, and laborious key-in method. Although more than half of the participants expressed their preference for print-based novels, the majority of them applauded the multiple functions of the Kindle reader and the advantage of its use in learning English. Several participants affirmed the Kindle reader's portability, capacity for storing many books, and interactive potential.

Similar findings were found when asked if the participants considered buying a Kindle reader a waste of money. Despite the fact that most participants preferred to read from print-based novels than from the Kindle, 61 percent of the participants disagreed that acquiring a Kindle reader was a waste of money. Common explanations include the Kindle's special functions and advantages, its projected popularity in the future, its cost-saving nature in buying many books, and its huge capacity for storing books. For instance, participant A said, "I think it will save money because I don't have to buy a lot of book[s]". Participant J expressed, "No, it is not a waste at all. Because I think the Kindle will be the trend in the future. Also, it is useful". Participant B revealed, "If you have a lot of books to carry, your back will hurt. By using the kindle, you just carry it and you can have those books handy. In addition, I think it will be a hit to read from the Kindle in few years". On the other hand, one third of those who deemed buying a Kindle a waste of money had their rationale. Their major reservation for buying a Kindle now has to do with its current market price. One participant protested, "Kindle is overpriced in Taiwan, and it is almost three times the price as it is in the States. I won't spend that much money. I might wait until it gets to a more reasonable price; then I will consider buying one. Besides, technologies evolve rapidly. The next version might be more user-friendly".

In addition to investigating the participants' preference over one mode of reading or the other, the researcher also probed into whether the medium of novel affects the participants' reading motivation. The majority of the participants do not see the medium of the novel as the primary factor accounting for their 
reading motivation. Instead, they regard the content and the plot of the novel as the major source of their reading motivation. For instance, Peter said, "I think the most important thing that affects me is the content of the books because they all have the same content, just one is paper and the other is an electronic reading machine". When asked if the medium of the novel affects their motivation to read, Joel from the Kindle group replied, "Not really, I read the novel simple because it is interesting to me. I think the content of the novel is much more important than the medium. It really affects my reading interest or reading motivation". In sum, the analysis of qualitative data from the participants suggests that the influence of reading mode on reading motivation is trivial. This finding is consistent with the independent samples t-test of the participants' post RMQ scores.

Different from what the researcher has anticipated, the findings from this study speak participants' discontentment with this relatively new reading gadget. Technology alone cannot convert a sluggish digital native reader into an avid one. In this case, medium does not matter as much as the sacred reading rituals deeply rooted in every experienced reader: the touch and the smell of the page. Books are here to stay until Kindle can thumb pages away.

\section{Implications and Conclusion}

Although more than half of the participants expressed their preference for print-based novels, most of them acknowledged the various functions of the Kindle reader and its potentiality for English language learning. Furthermore, the main reasons why the participants chose traditional books over the Kindle were either because of their static reading habits or their erroneous impression of the Kindle reader. Many participants saw the Kindle as very fragile. The participants' reaction to the incorporation of Kindle did not turn out as promising as the researcher had anticipated. This cohort of the adult EFL readers did not appreciate this new reading gadget. Instead, the technical aspects of the device hindered the full embracement from the digital native readers. Several participants complained that the button is hard to press for flipping pages, which created a barrier for uninterrupted reading.

In spite of the participants' dissatisfaction with the Kindle's incapability to flipping pages smoothly, the mean score from their post RMQ indicates four points increase in their overall motivation to read English novels. A slight decline was identified in the hard-copy group's post RMQ mean score. This difference suggests that the Kindle reader does boost up the participants' reading motivation. Reading via the Kindle provided motivation and situational interest and made reading more fulfilling (Chan \& Ahern, 1999). In other words, the Kindle facilitated the active engagement of the readers. Furthermore, a scrutiny into the 10 subcategories of reading motivation found a significant difference with the Kindle group in the pretest and the posttest of aesthetic enjoyment of reading. This finding points out that the Kindler has an upper hand in exposing readers to a variety of literary texts within one intact devise and subsequently enhanced their reading motivation. The use of technology not only has the potential to be a tool of great use in the language classroom, but also to serve as a vehicle for a richer reading experience and language learning. Though frustrations and difficulties were identified by the participants, they discovered the advantages of the Kindle reader and admitted that they do not like it because they were not used to using it yet. They have read hard copies of books for their entire life and were just starting to learn how to take advantage of this new reading device. Therefore, with the unstoppable trend of technological advancement in education, it is important for teachers to continuously explore meaningful ways of incorporating reading technology into their classrooms. 
It remains inconclusive as to whether integrating the newest technologies into the reading course will improve reading comprehension and boost reading motivation among EFL readers. The findings from the current study caution against adopting the newest electronic gadget into classroom practice before it is generally accepted in a given pedagogical context. For the future study of utilizing the Kindle or other electronic reading devices with the EFL readers, it is desirable to investigate its effects on actual reading behavior and the readers' interaction with the non-linear text. It will also be informative if the retention of medium-boosted, situational interest or motivation can be reexamined to gauge the sustainability of "motivational bounce" (Kamil et al., 2000). While the findings of this study are limited to the context in which the study took place, implications for classroom practices may be extended to other contexts. It is the researcher's hope that the teachers who seek to adopt electronic readers, such as the Kindle, do so with their unique needs and students in mind. The findings of this study support an emerging body of research stating that learning is socially constructed within the new literacies (Leu, Kinzer, Coiro, \& Cammack, 2004). Students clearly rely on each other for guidance, support, and construction of knowledge within the technology-rich environment, and so should educators.

\section{References}

Alexander, P. A., Kulikowich, J. M., \& Jetton, T. C. (1994). The role of subject-matter knowledge and interest in the processing of linear and non-linear text. Review of Educational Research, 64(2), 201-252.

Beck, I. L., \& McKeown, M. G. (1999). Comprehension: The sine qua non of reading. Teaching and Change, 6(2), 197-211.

Campbell, K. (2000). Gender and educational technologies: Relational frameworks for learning design. Journal of Educational Multimedia and Hypermedia, 9(2), 131-150.

Chan, T. S., \& Ahern, T. C. (1999). Targeting motivation: Adapting flow theory to instructional design. Journal of Educational Computing Research, 21(2), 151-163.

Chen, J., Warden, C. A., \& Chang, H. T. (2005). Motivators that do not motivate: The case of Chinese EFL learners and the influence of cultural on motivation. TESOL Quarterly, 39(4), 609-632.

Chu, M. L. L. (1995). Reader response to interactive computer books: Examining literary responses in a non-traditional reading setting. Reading Research and Instruction, 34(4), 352-366.

Crookes, G., \& Schmidt, R. (1991). Motivation: Reopening the research agenda. Language Learning, 41(4), 469-512.

Day, R., \& Bamford, J. (1998). Extensive reading in the second language classroom. Cambridge: Cambridge University Press.

Day, R., \& Bamford, J. (2002). Top ten principles for teaching extensive reading. Reading in a Foreign Language, 14(2). Retrieved February 20, 2009 from http://www.nflrc.hawaii.edu/rfl/October2002/day/day.html

Dörnyei, Z. (1990). Conceptualizing motivation in foreign-language learning. Language Learning, 40(1), 45-78.

Gardner, R. C. (1985). Social psychology and second language learning. London: Edward Arnold.

Grant, J. M. (2004). Are electronic books effective in teaching young children reading and comprehension? International Journal of Instructional Media, 31(3), 303-308.

Guthrie, J. T. (2001). Contexts for engagement and motivation in reading. Reading Online, 4(8). Retrieved February 10, 2009 from http://www.readingonline.org/articles/handbook/guthrie/

Guthrie, J. T., \& Wigfield, A. (2000). Engagement and motivation in reading. In M. L. Kamil, P. B. Mosenthal, P. D. Pearosn, and R. Barr (Eds.), Handbook of reading research: Vol. III (pp.403-422). Mahwah, NJ: Lawrence Erlbaum.

Hailey, D. E., \& Hailey, C. (1998). Hypermedia, multimedia, and reader cognition: An empirical study. Technical communication, 45(3), 330-342.

Harper, B., Squires, D., \& McDougall, A. (2000). Constructivist simulations: A new design paradigm. Journal of Educational Multimedia and Hypermedia, 9(2), 115-130.

Kamil, M. L., Intrator, S. M., \& Kim, H. S. (2000). The effects of other technologies on literacy and learning. In M. L. Kamil, P. B. Mosenthal, P. D. Pearson, and R. Barr (Eds.), Handbook of Reading Research (pp. 771-788). Mahwah, NJ: Erlbaum.

Kraemer, R. (1993). Social psychological factors related to the study of Arabic among Israeli high school students: A test of Gardner's socio-educational model. Studies in Second Language Acquisition, 15, 83-104. 
Labbo, L. D., Leu, D. J., Kinzer, C., Teale, W. H., Cammack, D., Soteriou, J. K., \& Sanny, R. (2003). Teacher wisdom stories: Cautions and recommendations for using computer-related technologies for literacy instruction. The Reading Teacher, 57(3), 300-304.

Larson, L. C. (2007). A case study exploring the "new literacies" during a fifth-grade electronic reading workshop (Doctoral dissertation, Kansas State University). Retrieved February 19, 2010 from krex.k-state.edu/dspace/handle/2097/352

Larson, L. C. (2008). Electronic reading workshop: Beyond books with new literacies and instructional technologies. Journal of Adolescent \& Adult Literacy, 52(2), 121-131.

Larson, L. C. (2009). E-reading and e-responding: New tools for the next generation of readers. Journal of Adolescent \& Adult Literacy, 53(3), 255-258.

Lefever-Davis, S., \& Pearman, C. J. (2005). Early readers and electronic texts: CD-ROM storybook features that influence reading behaviors. The Reading Teacher, 58(5), 446-454.

Leu, D. J. (2000). Exploring literacy on the Internet: Our children's future: Changing the focus of literacy and literacy instruction. The Reading Teacher, 53(5), 424-429.

Leu, D. J., Kinzer, C. K., Coiro, J., \& Cammack, D. W. (2004). Toward a theory of new literacies emerging from the Internet and other information and communication technologies. In R. B. Ruddell and N. Unrau (Eds.), Theoretical models and processes of reading (5th ed., pp. 1570-1613). Newark, DE: International Reading Association.

Lewin, C. (1996). Improving talking book software design: Emulating the supportive tutor. Bradford, England: Center for Information Technology in Education, The Open University.

Meyer, B. J. F., \& Poon, L. W. (1997). Age differences in efficiency of reading comprehension from printed versus computer-displayed text. Educational Gerontology, 23(8), 789-807.

Mori, S. (2002). Redefining motivation to read in a foreign language. Reading in a Foreign Language, 14, 91-110.

Pearman, C. J. (2008). Independent reading of CD-ROM storybooks: Measuring comprehension with oral retellings. The Reading Teacher, 61(8), 594-602.

Pearman, C. J., \& Lefever-Davis, S. (2006). Supporting the essential elements with CD-ROM storybooks. Reading Horizons, 46(4), 301-313.

Reinking, D. (1998). Synthesizing technological transformations of literacy in a post-typographical world. In D. Reinking, M. C. McKenna, L. D. Labbo, and R. D. Kieffer (Eds.), Handbook of literacy and technology: Transformations in a post-typographic world (pp. xi-xxx). Mahwah, NJ: Lawrence Erlbaum.

Takase, A. (2005). Japanese high school students' motivation for extensive reading. Reading in a Foreign Language, 19, 1-18.

Tompkins, G. E. (2006). Literacy for the 21st century: A balanced approach. Upper Saddle River, NJ: Pearson Prentice Hall.

Wishart, J. (2000). Students' and teachers' perceptions of motivation and learning through the use in schools of multimedia encyclopedias on CD-ROMs. Journal of Educational Multimedia and Hypermedia, 9(4), 333-347. 